\title{
HYPERPLASIA OF THYMIC GLAND: LEFT VIDEO-ASSISTED THORACOSCOPIC APPROACH
}

\section{Cerchia E., Bulotta A.L., Angotti R., Molinaro F., Di Maggio G., Messina M.}

\author{
Section of Pediatric Surgery, Department of Pediatrics, Obstetrics and Reproductive Medicine, \\ University of Siena, Siena, Italy
}

\begin{abstract}
Hyperplasia of thymic gland is a rare benign entity that should be considered in the differential diagnosis of anterior mediastinal masses in children and young adolescents. We report a case of a patient with a thymic mass, diagnosed occasionally for respiratory symptoms and treated by video-assisted thoracoscopic surgery. A previously healthy 10 years-old boy presented to our hospital for retrosternal pain and dyspnea with restriction to daily activities from four months. Diagnostic imaging was performed, including a chest x-ray and a magnetic resonance imaging, showing a large homogeneous anterosuperior mediastinal mass, more extended on the left side. The additional laboratory analysis, considered essential for differential diagnosis with myasthenia gravis and lymphoma, resulted negative. In view of these findings, our patient underwent to video assisted thoracoscopy with left-sided approach for a total resection of thymus and perithymic fat. The patient made an excellent recovery without postoperative complications and was discharged from the hospital four days later. Histopathological examination showed a normal thymic architecture like a true thymic hyperplasia. At follow up, chest x-ray was normal in absence of pleural and parenchimal alterations. Thoracoscopic thymectomy is a safe technique that allows to achieve the goal of early thymectomy with the advantages of less invasive procedure.
\end{abstract}

Key words: Anterior mediastinal masses, thymic hyperplasia, video-assisted thoracoscopy.

\section{INTRODUCTION}

Thymic enlargement in childhood may be ascribed to various causes including thymoma, thymolipoma, lymphoid follicular hyperplasia and benign thymic hyperplasia. This latter disorder is considered rare with few cases described in the world literature [1]. True thymic hyperplasia corresponds to a thymic enlargement due to a generalized increase in number of thymic cells without alteration of normal architecture and histology [2]. Although rare, true thymic hyperplasia should be considered in the differential diagnosis with others anterior mediastinal masses in children and young adolescents. These lesions infrequently can cause acute respiratory symptoms that are related to compression of adjacent structures. In these cases, a surgical management has been advocated as a definitive therapeutic option. Conventionally, a trans-sternal approach is used for thymectomy since the advent of the minimally invasive surgical technique, a video-assisted thoracoscopic approach has been reported in order to obtain a less invasive access and excellent cosmetic results [3-4]. We report a case of a patient with a thymic mass, diagnosed occasionally for presence of respiratory symptoms, who was treated by video assisted thoracoscopic surgery.

\section{CASE REPORT}

A 10 years-old boy presented to our hospital for several episodes of dyspnea after physical practice and retrosternal pain with restriction of daily activities from four months. Physical examination revealed an healthy boy weighting $40 \mathrm{~kg}$ with normal vital signs. Diagnostic imaging was performed including a chest $\mathrm{x}$-ray and magnetic resonance imaging (MRI), revealing a large homogeneous left-sided mediastinal mass contiguous to the anterior mediastinum (Figure $1 \mathrm{~A}$ and $\mathrm{B}$ ). The additional laboratory analysis, considered essential for differential diagnosis with myasthenia gravis and lymphoma, resulted negative. In view of these findings, we submitted our patient to a video assisted thoracoscopy with left-sided approach to facilitate the dissection of both thymus and perithymic fatty tissue.

\section{Surgical technique}

The technique is performed, under general anesthesia, from the left side with the patient in the $30^{\circ}$ right lateral decubits position under single right lung ventilation. Three trocars of $5 \mathrm{~mm}$ are placed at the fifth intercostal space of the left anterior axillary line for introduce a $30^{\circ}$ telescope, at the third and seventh intercostal space of

\section{Correspondence to:}

Mario Messina

Division of Pediatric Surgery, Dept of Pediatrics, Obstetrics and Reproductive Medicine.

University of Siena. Policlinico "Le Scotte", Viale Bracci 53100, Siena, Italy.

Telefono: +39577586501, Fax :+39577586174

E-mail: mario.messina@unisi.it 
the midaxillary for instruments. After general exploration, the dissection was begun caudally along the capsule of the thymus gland with progressive exsposure of the lower pole by using harmonic scalpel. Lateral dissection included pericardial tissue preserving the phrenic nerve and proceeding towards the innominate vein. We continued to dissect the thymic mass, from the right parietal pleura and the left perithymic fat, cranially until the mammarian artery and vein (Figure 3). At the end resected gland was brought into an endo-bag. After control of hemostasis, a drainage tube was placed in the seventh intercostal space.

\section{RESULTS}

The procedure was well tolerated with a mean operation time of $90 \mathrm{~min}$ and without complication, except for a minimal controlled bleeding. The oral feeding was restored on the first postoperative day and the drainage was removed in the second postoperative day. The patient made an excellent recovery and was discharged from the hospital four days later, after a negative radiological control (Figure 4). Histopathological examination showed thymic tissue of $60 \mathrm{~g}$ with a preservation of normal architecture compatible with a true thymic hyperplasia (Figure 5). At follow up the patient was totally asymptomatic during daily activities with a good cosmetic result.

\section{DISCUSSION}

The thymus is embriologically derived from the third and fourth pairs of pharyngeal pouches. At birth, it weights from 10 to $35 \mathrm{~g}$ and continues to grow in size until puberty, when it achieves a maximum weight of 10 to $50 \mathrm{~g}$, then it undergoes to progressive atrophy [5].
The thymus gland increases in size without a known etiology is defined benign thymic hyperplasia. Histologically, two types of thymic hyperplasia are recognized: the first, "true hyperplasia", is defined as an increase in both size and weight of the gland with retention of a normal architecture as in our case; the second type, "lymphoid hyperplasia", is characterized by the presence of lymphoid follicles with germinal centers, usually not accompanied by a marked increase in thymic size [6]. Clinically, thymic hyperplasia can mimic others important diseases, including thymoma, lypoma and germ cell tumors. Thymic enlargement is generally asymptomatic, but when respiratory symptoms are present, they may influence the management [7]. In these cases, most of authors support surgical management as the best therapeutic option [8-9]. Several surgical approaches to thymectomy exist, the thoracoscopic one was first reported by Sugarbaker from Boston and also the Belgium group in 1993 [10-11]. After, it has evolved with several variants including video-assisted thoracic surgery (VATS) unilateral thymectomy through three ports, the bilateral thoracoscopic approach combined with a cervical incision for extended thymectomy and more recently endoscopic robot-assisted thymecthomy. Minimally invasive techniques have become popular due to their low procedural mortality and morbility associated to minimal surgical trauma with the best cosmetic results. Yim et al. first proposed a right-sided approach for VATS thymectomy justifying this for the greater maneuverability of instruments in the wider right pleural cavity and easier identification of the left innominate vein using superior vena cava as a landmark [12]. Mineo et al. advocated a left-sided approach because the dissection maneuvers are safer for the position of the superior vena cava out of the surgical field with less risk of accidental injury [13]. In addition, the resection of perithymic fatty tissue around the left pericardio-
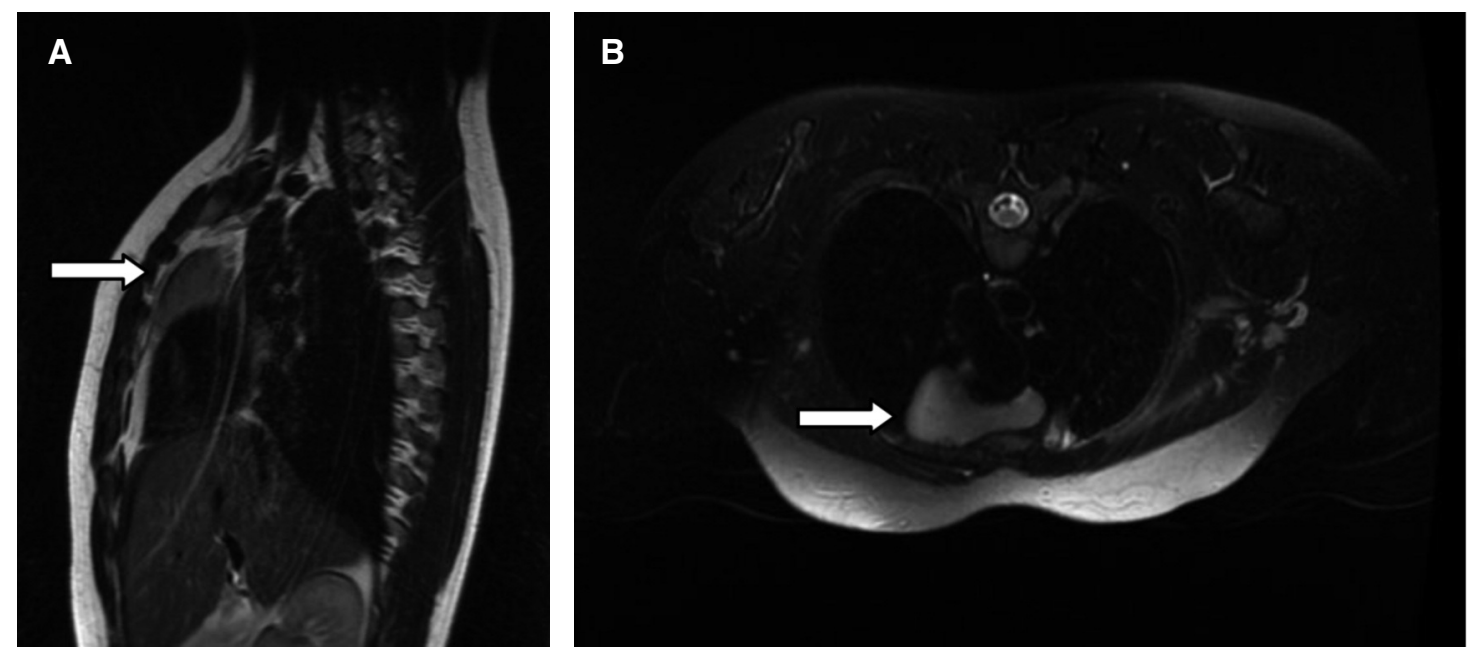

Figure 1. A and B. MRI study shows a large antero-superior mediatinal mass left-sided (arrows). 


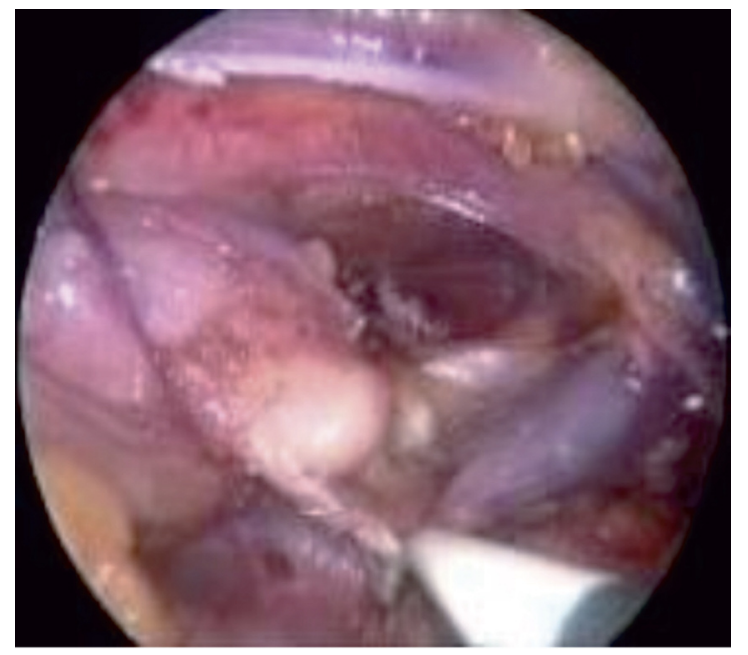

Figure 3. Thoracoscopic isolation of upper thymic pole.

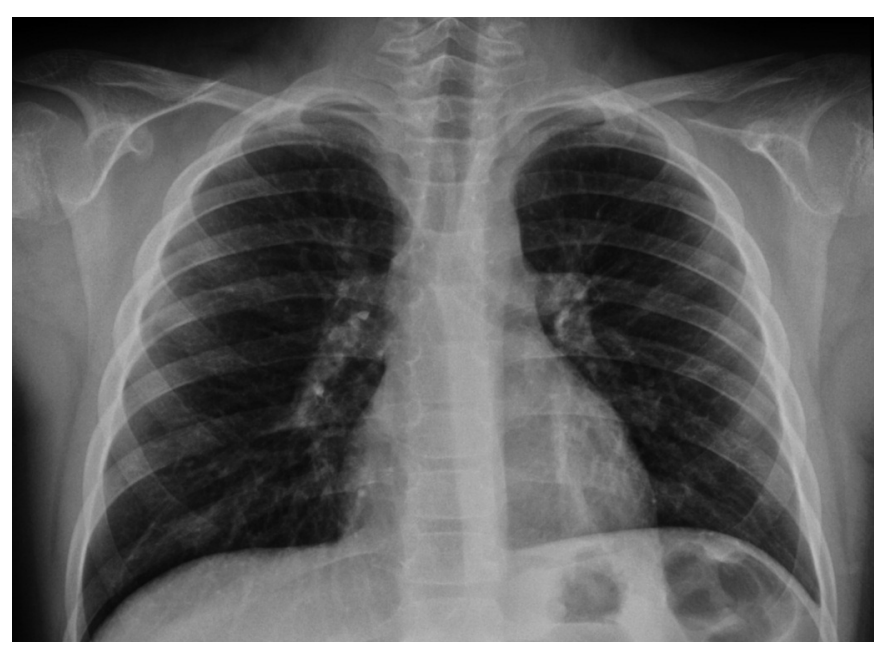

Figure 4. Chest X-ray at post operative control.

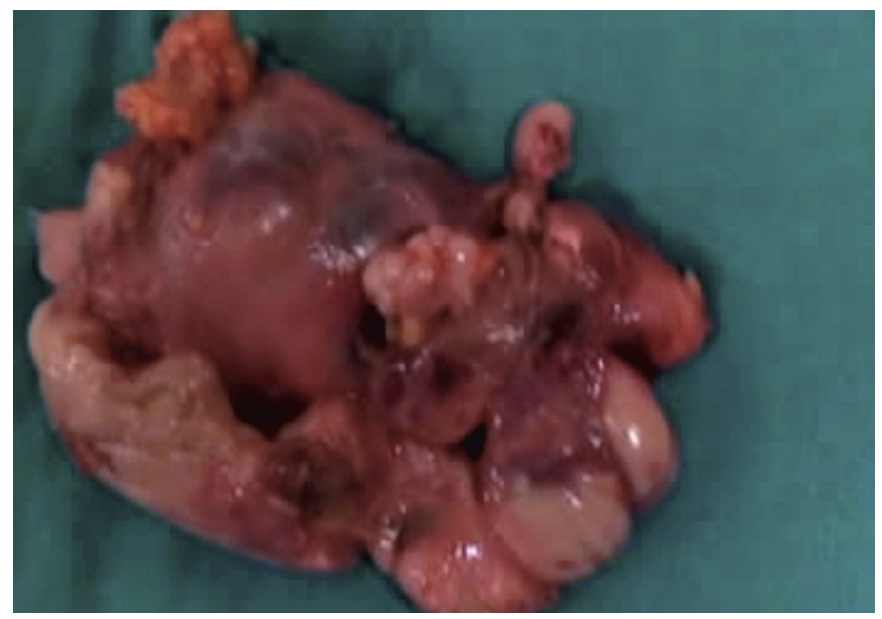

Figure 5. Hyperplastic thymic gland with perithymic fat. phrenic angle and aortopulmonary window can be performed more readly from the left side. Even if the thymus can be completely removed using a left or right approach, in our case we chose the left access because the thymic gland was larger in the left side and to allow an extended resection of potential ectopic thymic tissue including the aortopulmonary window. Videoassisted thoracoscopic thymectomy is a successful technique in experienced hands and represents an increasingly popular alternative approach to conventional open techniques because it allows to achieve the goal of early thymectomy with the advantages of less invasive procedure.

\section{REFERENCES}

1. Ocal T., Turken A., Ciftci A., et al. Thymic enlargement in childhood. Turk J Pediatric 2000; 42:298-303.

2. Ricci C, Pescarmona E., Rendina EA, et al. True thymic hyperplasia: a clinicalpathological study. Ann Thorac Surg 1989; 47:741-5.

3. Linegar AG., Odell JA., Fennel WMP, et al. Massive thymic hyperplasia. Ann Thorac Surg 1991;57:21-3.

4. Yim AP., Izzat MB. VATS approach to the thymus. Minimal Access Cardiothoracic Surgery 2000;209-20.

5. Rice HE., Flake AW., Hori T., et al. Massive thymic hyperplasia: carachterization of a rare mediastinal mass. J Ped Surg 1994;29:1561-4.

6. Zheng T., Li-Yang Y., et al. True thymic hyperplasia in an infant. J Ped Surg 2010; 45:1711-1713.

7. Szarf G., De Andrade M, De Oliveira R., et al. Massive thymic hyperplasia presenting with respiratory insufficiency in a 2-year-old child. Thorax 2010; 65:555-556.

8. Cheuk W., Tsang W.Y., Chan J.K. Microthymoma: definition of the entity and distinction from nodular hyperplasia of the thymic epithelium (so-called microscopic thymoma). Am Surg Pathol 2005; 29:415-419.

9. Landrenau RJ., Dowling RD., Castillo WM. Thoracoscopic resection of an anterior mediastinal tumor. Ann Thorac Surg 1992; 54:142-4.

10. Sugarbaker DJ. Thoracoscopy in the management of anterior mediastinal masses. Ann Thorac Surg 1993;56:653-6.

11. Coosemans W., Lerut TE, Van Raemdonck DE. Thoracoscopic surgery: the Belgian experience. Ann Thorac Surg 1993;56:721-30.

12. Ng CS., Wan IY., Yim AP. Video-assisted thoracic surgery thimectomy: the better approach. Ann Thorac Surg 2010; 6:2135-2141.

13. Mineo TC, Pompeo E., Lerut TE., Bernardi G, Coosemans W., Nofroni I. Thoracoscopic thymectomy in autoimmune myasthenia gravis: results of left sided approach. Ann Thorac Surg 2000;69:1537-41. 